\title{
Efectos de cambios regulatorios sobre el desempeño del pool eléctrico español entre 2004 y 2006
}

\author{
Recibido: noviembre 10, 2016 - Aceptado: septiembre 26, 2017
}

Doi: http:/ /dx.doi.org/10.12804/revistas.urosario.edu.co/economia/a.6456

\author{
John Jairo García Rendón* \\ Jhonny Moncada Mesa ${ }^{+}$
}

\section{Resumen}

Este trabajo examina la relación entre el Gap del precio marginal del sistema y otras variables típicas del mercado, organización industrial y cambios regulatorios en el pool eléctrico español. Se estima un modelo de panel de datos largo, en el cual la dimensión temporal es mayor al número de empresas consideradas. Los resultados indican que tanto la alta concentración del mercado como la posibilidad de que las empresas más grandes sean pivotes generaron distorsiones sobre la determinación del precio. Además, los cambios regulatorios implementados por las autoridades no indujeron los efectos esperados, lo que crea distorsión e incentivos incorrectos en este mercado.

Palabras clave: regulación, precios, cointegración en panel, pool eléctrico español. Clasificación JEL: L51, L13, C23

Esta investigación fue financiada por el Departamento Administrativo de Ciencias, Tecnología e Innovación (Colciencias) mediante su programa de Jóvenes Investigadores y la Universidad EAFIT.

* Escuela de Economía y Finanzas, Universidad EAfIT. Correspondencia: Dirección: Carrera 49 \# 7Sur-50, bloque 26. Teléfono: (+57 4) 2619500 ext. 9009 . Correo electrónico: jgarcia@eafit.edu.co

† Escuela de Economía y Finanzas, Universidad EAFIT. Correo electrónico:jmoncad7@ eafit.edu.co

Cómo citar este artículo: García-Rendón, J. J., \& Moncada-Mesa, J. (2017). Efectos de cambios regulatorios sobre el desempeño del pool eléctrico español entre 2004 y 2006. Revista de Economía del Rosario, 20(2), 243-273.

Doi: http://dx.doi.org/10.12804/revistas.urosario.edu.co/economia/a.6456 


\title{
Regulatory Changes and their Effects on the Performance of the Spanish Electricity Pool in 2004-2006
}

\begin{abstract}
This paper examines the relation between the Gap of the marginal price in the Spanish electricity market and other variables, the industrial organization of this market, and key regulatory changes affecting the market. We estimate a panel model with a large time series dimension in a small cross section of firms. We find that, due to high market concentration, the biggest firms are pivotal in the determination of market prices, distorting them and distancing them from competitive prices. The regulatory changes implemented did not have their intended effects, but rather created more distortions and perverse incentives in the market.
\end{abstract}

Keywords: Regulation, prices, panel co-integration, Spanish electricity pool. JEL Classification: L51, L13, C23

\section{Efeitos de mudanças regulatórias sobre o desempenho do pool elétrico espanhol entre 2004 e 2006}

\section{Resumo}

Este trabalho examina a relação entre o Gap do preço marginal do sistema e outras variáveis típicas do mercado, organização industrial e mudanças regulatórias no pool elétrico espanhol. Se estima um modelo de painel de dados longe, no qual a dimensão temporal é maior ao número de empresas consideradas. Os resultados indicam que tanto a alta concentração do mercado quanto a possibilidade de que as empresas mais grandes sejam pivôs geraram distorções sobre a determinação do preço. Além disso, as mudanças regulatórias implementadas pelas autoridades não induziram os efeitos esperados, criando distorção e incentivos incorretos neste mercado.

Palavras-chave: regulação, preços, cointegração em painel, pool elétrico espanhol. Classificação JEL: L51, L13, C23 


\section{Introducción}

Desde la entrada en vigencia de la Directiva 96/92/CE de diciembre de 1996, en la que se establecen normas comunes para el mercado interior de la electricidad, destinada a abrir el mercado a la competencia, y la Directiva 98/30/ CE de julio de 1998, concerniente a las reglas comunes para el funcionamiento interior del mercado del gas, para el sector eléctrico y del gas respectivamente, empieza el esfuerzo gradual de liberación de la industria energética en Europa. Esto llevó particularmente, en el caso de España, a un proceso de liberación en el mercado eléctrico, que en sus inicios mantuvo niveles bajos en el precio a pesar de que paralelamente se dio un fenómeno de alta concentración (Kuhn \& Machado, 2004; Fabra \& Toro, 2005; Pérez, Batlle, Vázquez, Rivier, \& Rodilla, 2005). Sin embargo, desde 2004 se inició un incremento acelerado en el precio que llegó a su punto máximo a mediados de 2005. Todo esto produjo que las autoridades regulatorias iniciaran un conjunto de cambios, entre los que fueron los más representativos para el periodo 2004-2006 el Real Decreto Ley (RDL) 5/2005 y RDL 7/2006 concernientes al desmonte de los Costos de Transición a la Competencia (CTC) y el RDL 3/2006, que cambia el modo de casación de ofertas y demandas en el sistema.

No obstante, si bien el objetivo de la regulación era mantener precios bajos al igual que en los primeros años de la liberación, los resultados no fueron los esperados. Las tarifas residenciales para la prestación del servicio de electricidad para el año 2012 son las más altas, 24,7 \$US Cent / kWh, al compararse con el resto de países de la OCDE (ECSIM, 2013; IEA, 2013). Entre las causas argumentadas por los autores se encuentra cómo el mecanismo de СтС, que venía siendo implícitamente un price cap, ya no tenía el efecto esperado en los agentes. Además, el cambio en la casación no generaba en el mercado lo mismo que una medida de contratos bilaterales. Por tanto, España, al verse motivado a realizar los cambios necesarios para mejorar el funcionamiento del mercado, no lo planeó debidamente, lo que ha llevado a que en la actualidad sufra un déficit tarifario.

Esta investigación estudia el problema presentado en el mercado de generación de energía eléctrica español entre 2004 y 2006, tiempo en el cual se comenzaron a evidenciar los graves problemas que persisten en el mercado. Por tanto, este trabajo pretende responder a la siguiente pregunta de investigación: ¿Cuáles fueron los efectos de los sucesivos cambios regulatorios en el mercado de electricidad de España en el periodo 2004-2006 sobre el precio marginal del sistema y, por lo tanto, en el funcionamiento del mercado? Es importante anotar que los cambios regulatorios realizados durante este 
periodo no han sido estudiados en profundidad y, por ello, esta es la contribución de este estudio.

Para dar respuesta a esta pregunta, se realiza una estimación del Gap del precio marginal del sistema (PMS), teniendo en consideración los efectos de los cambios regulatorios presentados en el mercado. Para esto se utiliza una metodología en dos fases, en la primera, por medio de la organización industrial, se calculan índices como el Residual Supply Index (RSI) y HerfindahlHirschman Index (HHI), que permiten tener una panorámica general relacionada con el poder de mercado y la concentración en esta industria. En la segunda, se estima un macro panel que explica el Gap del PMS, en función de ciertas variables relevantes para el mercado y dummies que recogen los efectos de la estructura regulatoria.

El texto, luego de la introducción, se divide en cinco partes. En la primera se describe brevemente el funcionamiento del sistema, donde se presenta la situación general del mercado para el periodo de estudio. En la segunda se muestra el estado del arte con la discusión actual sobre el mercado, finalizando la sección con el planteamiento de la hipótesis a probar. En la tercera parte se muestra la metodología, diferenciando la parte descriptiva de la estimación econométrica. En la cuarta parte se esbozan los resultados más relevantes. Finalmente, se realizan algunas conclusiones.

\section{Funcionamiento del mercado de energía eléctrica en España}

En 1998, mediante la Ley 54/1997, del sector eléctrico, empieza a funcionar el Pool eléctrico español. Los objetivos básicos de la política energética debían ser consistentes en hacer compatible la progresiva liberalización del mercado y garantizar el suministro eléctrico y la calidad de dicho suministro al menor costo posible. Para lograr tal fin, se crea un mercado diario donde, igual que funciona en la gran mayoría de los mercados internacionales, incluido Colombia, el operador del mercado (OMIE en la actualidad, OMEL en su momento) es el encargado de realizar las casaciones horarias para determinar el precio. Después de que los generadores realizan sus ofertas diarias de cantidades y precios para cada hora del día siguiente, el operador las cruza para cada hora con el pronóstico de demanda y el precio resultante de este proceso, que corresponde al último precio que se ofertó necesario para satisfacer la demanda, conocido como el precio marginal del sistema, es el que se le remunera a cada uno de los generadores que son necesarios para abastecer la demanda. Este mercado funciona como una subasta de precio uniforme y los precios se determinan por orden de mérito de oferta, es decir, las más baratas. 
También se crea una serie de mercados intradiarios y de servicios auxiliares, lo que da origen a los mercados intradía, como se conocen a nivel mundial, con el fin de solucionar las restricciones técnicas que se pueden presentar en el sistema para garantizar la seguridad y la calidad del suministro de energía en tiempo real.

Tabla 1. Energía generada por tecnología en España

\begin{tabular}{lrrrrrr}
\hline \multirow{2}{*}{ Tecnología / Producción } & \multicolumn{2}{c}{2004} & \multicolumn{2}{c}{2005} & \multicolumn{2}{c}{2006} \\
\cline { 2 - 7 } RWh & \multicolumn{1}{c}{$\%$} & GWh & \multicolumn{1}{c}{$\%$} & \multicolumn{1}{c}{ GWh } & \multicolumn{1}{c}{$\%$} \\
\hline Régimen ordinario & 206412 & 81,49 & 212955 & 80,47 & 220933 & 80,93 \\
Hidráulica & 29777 & 11,76 & 19170 & 7,24 & 25330 & 9,28 \\
Nuclear & 63606 & 25,11 & 57539 & 21,74 & 60126 & 22,02 \\
Carbón & 76358 & 30,14 & 77393 & 29,24 & 66066 & 24,20 \\
Fuel-Gas & 7697 & 3,04 & 10013 & 3,78 & 5905 & 2,16 \\
Ciclo combinado & 28974 & 11,44 & 48840 & 18,45 & 63506 & 23,26 \\
Régimen especial & 46894 & 18,51 & 51690 & 19,53 & 52071 & 19,07 \\
Cogeneración & 19267 & 7,61 & 18736 & 7,08 & 16655 & 6,10 \\
Eólica & 16087 & 6,35 & 21190 & 8,01 & 23188 & 8,49 \\
Solar & 18 & 0,01 & 41 & 0,02 & 106 & 0,04 \\
Hidráulica & 4758 & 1,88 & 3816 & 1,44 & 4144 & 1,52 \\
Biomasa & 1761 & 0,70 & 2121 & 0,80 & 2144 & 0,79 \\
Residuos & 2291 & 0,90 & 2608 & 0,99 & 2442 & 0,89 \\
Tratamiento residuos & 2712 & 1,07 & 3178 & 1,20 & 3392 & 1,24 \\
Total & 253306 & 100,00 & 264645 & 100,00 & 273004 & 100,00 \\
\hline
\end{tabular}

Fuente: elaboración propia a partir de datos de OMEL y OMIE.

En la tabla 1 se muestra la energía generada por cada una de las fuentes para los dos regímenes que conforman el pool, el régimen ordinario y el especial, para el periodo entre 2004 y 2006. El Régimen Ordinario de acuerdo con la Ley 54/1997 está compuesto por fuentes de generación de carbón, fuel-oil, centrales nucleares, ciclos combinados de gas y grandes centrales con más de 50 MW. Por su parte, el especial lo conforman las plantas de generación con una potencia instalada menor a $50 \mathrm{MW}$ que utilicen la cogeneración, energías renovables y biomasa, entre otros. Como se puede observar, el régimen ordinario aportó el $80 \%$ de la generación al sistema en 2006, donde las tecnologías a carbón $(24,20 \%)$, ciclo combinado $(23,26 \%)$ y nuclear $(22,02 \%)$, en orden 
de importancia, fueron las que más aportaron al sistema y, en el especial, la eólica presentó su mayor participación (8,49\%).

Varios aspectos han generado problemas en el funcionamiento del Pool, entre ellos lo que se conoce en la actualidad como el déficit del sistema español de energía eléctrica. Estos problemas tuvieron su origen desde la concepción inicial de la estructura del mercado. Sin embargo, es desde el periodo 20042006 donde se hacen evidentes los problemas y se empiezan a implementar las medidas para corregirlos, pero estas al parecer no han tenido los efectos esperados.

Son varios los factores que han afectado el funcionamiento del mercado. La contratación bilateral física está prevista en la Ley, pero por motivos, entre ellos, de deficiencia en la normativa que solo hasta el 2004 se superaron (Pérez et al., 2005), se generó que, en la práctica, el mercado mayorista funcionara como un mercado de corto plazo. Solo hasta marzo de 2006, con la medida transitoria establecida en el RDL 3/2006, que asimila los contratos bilaterales de las energías presentadas simultáneamente al mercado diario e intradiario por agentes al sector eléctrico pertenecientes al mismo grupo empresarial. De manera que estos solo pueden participar en la casación neta del grupo, que podrá ser alternativamente compradora o vendedora. Con el RD 1634/2006, que introduce las subastas vPP (Virtual Power Plant, ventas virtuales de energía) para Endesa e Iberdrola, principales empresas del sector. Y en el 2007, por medio de la Orden ITC/400/2007, por la que se regulan los contratos bilaterales que firmaban las empresas distribuidoras, se empieza a negociar por medio de los contratos bilaterales una cantidad mayor.

Aunque estas medidas disminuyeron en cierto nivel el poder de mercado, como estaba previsto en uno de sus objetivos, aún el volumen y el plazo de estos contratos no ha sido lo suficientemente grande y extenso, respectivamente, para mitigar considerablemente dicho poder (de Frutos \& Fabra, 2008; Federico, Vives \& Fabra, 2008).

A pesar de que en los primeros años de la desregulación del sector eléctrico en España las dos empresas más grandes, Endesa e Iberdrola, controlaban conjuntamente el $80 \%$ de la generación total de la industria; el asunto de los precios spot no fue de gran preocupación para los encargados de la regulación. Esto debido al mecanismo asumido para la recuperación de los Costes de Transición a la Competencia (СтC), el cual establecía un price cap en el mercado (Ciarreta \& Espinosa, 2012), lo que llevó a un nivel de precios bajos en los primeros años (Fabra \& Toro, 2005; Kuhn \& Machado, 2004; Pérez et al., 2005). Así, dada la estructura de integración vertical que tenían las dos principales empresas, se condicionaban los incentivos a fijar un precio alto/ 
bajo según su posición de compradores o vendedores en el mercado (Agosti, Padilla \& Requejo, 2007).

Sin embargo, a partir de 2004, con la pérdida de credibilidad respecto al mecanismo de CTC, mediante el RDL 5/2005, que aplaza la liquidación definitiva de los CTC correspondientes al año 2004, hasta el año 2006, se evidencia la falta de eficacia del mecanismo de CTC en el mediano plazo. Al mismo tiempo que las empresas que como en el caso de Iberdrola habían recuperado, durante los primeros años, una gran parte de su asignación inicial de los CTC y, a su vez, entraron nuevos generadores al mercado sin derecho a los СТС. Esto llevó a que el mecanismo para mantener un nivel de precios bajo en el mercado spot perdiera credibilidad. Además de dejar clara la incertidumbre reguladora para la asignación de los CTC (Federico et al., 2008; Pérez et al., 2005) y, que los precios hayan tenido grandes aumentos como el que se presentó en el 2005, prácticamente el doble frente al 2004, y que se sostuvo en el 2006.

Esto hace interesante analizar el poder de mercado en el mercado diario de generación eléctrica en España durante el 2004-2006 y evaluar el efecto que ha tenido la implementación de los cambios en el funcionamiento de este mercado, cuyo objetivo se propone este escrito. Por lo tanto, tal y como lo exponen Gianfreda y Grossi (2012), este tipo de trabajos tienen su fundamento en diversos aspectos que competen tanto al mercado como a la sociedad en general.

\section{Estado del arte}

Son varios los trabajos que tratan el comportamiento del mercado de generación eléctrica, entre los principales estudios que se han realizado y que hacen alusión a este tema para el Pool eléctrico español, algunos han utilizado indicadores básicos como el Índice Herfindahl-Hirschman (HHI), el índice de suministradores pivote (ISP) (CNE, 2005, 2007; Federico et al., 2008), o el índice de Lerner (Moutinho, Moreira \& Mota, 2014). Otros han adoptado el enfoque utilizado por von der Fehr y Harbord (1993) sobre multi-unit auction (de Frutos \& Fabra, 2008; García-Díaz \& Marín, 2003). También el modelo de Cournot se ha empleado para tal fin (Fabra \& Toro, 2005; Ocaña \& Romero, 1998; Pérez Arriaga et al., 2005).

Federico et al. (2008) para el periodo entre el 2004-2007, por medio del HHI, para el régimen ordinario en España encuentran, considerando los dos umbrales definidos, el de la Comisión Europea $(2,000)$ o la Comisión Federal de la Energía de los Estados Unidos $(1,800)$, que se trata de un mercado altamente concentrado. Y por medio del IsP para las dos empresas más grandes, 
Iberdrola y Endesa, en 2006 y 2007, encuentran que Iberdrola era pivote para cerca del $10 \%$ y Endesa 6,5\% en el primer año. Además de mostrar que, a pesar de que los niveles de pivotalidad en el 2007 disminuyen considerablemente frente al 2006 para ambas empresas, por medio del Residual Supply Index (RSI), confirman que conjuntamente siguen siendo pivotes para una gran parte de horas durante este año. También la CNE (2005) y el TDC ${ }^{1}$ (2005) utilizaron estos dos indicadores para el proyecto de concentración consistente en la adquisición del control de Endesa por parte de gas natural, encontrando para el periodo 2004-2006 altos niveles de concentración para el régimen ordinario.

Por su parte, Moutinho et al. (2014) para los periodos 2002-2005 y 2006-2007 encuentran que las medidas de poder de mercado adoptadas, como el índice de Lerner, tienen un impacto sobre las estrategias de los precios de oferta y las cantidades netas que presentan las empresas en el mercado. Además, los incentivos implantados a las empresas, como el price cap (СтС) en el contexto de transición a la competencia, resultó — para el primer periodo estudiado en unos precios bajos- que se diluyó y luego generó de nuevo un aumento, lo que va en línea con Ciarreta y Espinosa (2012), y lo que encuentran Pérez Arriaga et al. (2005) desde mediados de 2004, quienes determinan que figura "la existencia de un nivel inaceptable de poder de mercado en el sistema eléctrico español contemplando tanto su estructura actual, como lo que se asegura en el mediano plazo". Finalmente, evidencian que debido a la excesiva intervención regulatoria ocurrida desde 2006 se han generado la crisis financiera (déficit tarifario) y la dramática caída en la inversión y concluyen que se hacen necesarios más trabajos sobre los efectos de estas políticas en el mercado.

De Frutos y Fabra (2008) establecen que la efectividad del establecimiento de los contratos forward en la mitigación del poder del mercado depende tanto del volumen del contrato como de la distribución entre las empresas. Para el caso español realizan estimaciones para el volumen asignado de VPP y encuentran alto poder de mercado. García-Díaz y Marín (2003) encuentran que dicho poder aumentó substancialmente como efecto de la fusión de 1996 que transformó la industria de una estructura de seis empresas a cuatro, lo que sugiere que las asimetrías en tamaños y costes de las empresas generadoras desempeñan un papel crucial en la determinación de los precios, ya que llevan a márgenes más altos de beneficios por parte de las empresas grandes. Mientras que las pequeñas son tomadoras de precios. Además, al comparar los resultados obtenidos por medio del enfoque de subasta con el del

1 Tribunal de Defensa de la Competencia, "Resolución, Expediente 580/04, Gas Natural", junio de 2005. En la actualidad, la TDC para a ser la Comisión Nacional de la Competencia CNC. 
modelo de Cournot, encuentran que con el último se obtienen precios más altos frente al primer enfoque, lo que coincide con los resultados encontrados por Green y Newbery (1992) para el mercado spot de generación inglés. Por su parte, Fabra y Toro (2005) muestran que el desempeño del pool eléctrico español durante el primer año de funcionamiento, 1998, no es consistente con las predicciones de los modelos de comportamiento individual de maximización de beneficios, pudiendo existir una colusión tácita. Kühn y Machado (2004) utilizan el modelo de función de oferta para analizar el poder de mercado en precios y eficiencia productiva para el mismo pool en el 2001. Sus resultados sugieren que el poder de mercado presentó poco impacto en los precios del mercado spot español, pero que las empresas pudieron haber presentado substanciales ineficiencias productivas.

Como se puede observar, son varios los trabajos realizados para el estudio del funcionamiento y el comportamiento del poder de mercado. No obstante, esta propuesta pone su foco de atención en los efectos de los sucesivos cambios regulatorios, que no han tenido los efectos esperados, especialmente en contrarrestar el poder de mercado, lo que ha desencadenado en un aumento importante del precio spot de generación eléctrica en el periodo propuesto. Son varios factores que hacen visible esta situación, el planteamiento inicial de un sistema que genera incentivos para un funcionamiento de corto plazo, la alta concentración del mercado y los sucesivos cambios regulatorios no están generando los incentivos necesarios para un correcto funcionamiento del mercado.

\section{Metodología y datos ${ }^{2}$}

La metodología utilizada en este trabajo está dividida en dos partes. En la primera, se calculan índices de concentración y de poder de mercado para caracterizar el comportamiento del sector. En la segunda, se usa la regresión econométrica para testear mediante la evidencia empírica las relaciones entre cada una de las variables y el Gap del precio marginal del sistema. A continuación, se presentan cada una de las metodologías.

\subsection{Cálculo de indices}

En la tabla 2 se presentan los índices Herfindahl-Hirschman y Residual Supply Index, los cuales se utilizan para el análisis del funcionamiento del mercado y que dará una visión clara de cuál es su situación en el periodo estudiado.

2 La presentación aquí realizada se basa en los trabajos de Moutinho, et al. (2014) y Blackburne, et al. (2007). 
Tabla 2. Índices de Concentración y poder de mercado

\begin{tabular}{|c|c|c|}
\hline Nombre & Cálculo & Concepto \\
\hline 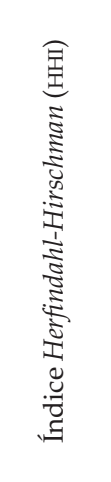 & $\begin{array}{l}\text { Se define como la suma de } \\
\text { los cuadrados de la parti- } \\
\text { cipación en el mercado de } \\
\text { la firma i en la industria }{ }^{3} \text {. } \\
\text { Su estimación se hace co- } \\
\text { mo lo muestra la siguiente } \\
\text { ecuación: } \\
H H I=\sum_{i=1}^{N} \mathrm{~S}_{i}^{2} \\
\text { donde Ses la participación } \\
\text { en el mercado. }\end{array}$ & $\begin{array}{l}\text { Cuanto mayor sea la participación de una empresa en el } \\
\text { mercado, mayor será su interés para que los precios que } \\
\text { remuneran el total de su producción sean elevados. Este } \\
\text { índice toma en cuenta el número de firmas y las desigual- } \\
\text { dades en la participación en un mercado determinado. } \\
\text { El valor del HHI va de 0, donde la industria actúa bajo un } \\
\text { esquema de competencia perfecta, a 10000, donde sucede el } \\
\text { caso opuesto; es decir, una estructura de monopolio puro. } \\
\text { Este índice ha sido una de las medidas tradicionales en que } \\
\text { se apoyan las instituciones de defensa de la competencia } \\
\text { para determinar el grado de competencia en una industria } \\
\text { o mercado como una primera aproximación a la capacidad } \\
\text { efectiva y a los incentivos económicos de un agente para } \\
\text { ejercer poder de mercado }\end{array}$ \\
\hline 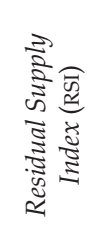 & $\begin{array}{l}\mathrm{RSI}_{\mathrm{i}}=\text { (Potencia total dispo- } \\
\text { nible - Potencia relevante } \\
\text { de la empresa i) / la de- } \\
\text { manda de energía total (es } \\
\text { decir, el cociente entre la } \\
\text { oferta residual de una em- } \\
\text { presa y la demanda total). }\end{array}$ & $\begin{array}{l}\text { Un valor de RSI mayor a } 100 \% \text { en una hora específica sig- } \\
\text { nifica que el generador i tiene poca capacidad para afectar } \\
\text { el precio. Los otros generadores tendrían potencia suficiente } \\
\text { para abastecer la demanda. Por el contrario, un valor de RSI, } \\
\text { por debajo del } 100 \% \text { refleja la existencia de cierto potencial } \\
\text { para ejercer poder de mercado, debido a que la potencia del } \\
\text { generador i es necesaria para abastecer la demanda. }\end{array}$ \\
\hline
\end{tabular}

Fuente: elaboración propia.

\subsection{Estimación econométrica}

La segunda parte de la metodología comprende la estimación de un modelo econométrico por medio de un macro panel. En este caso, basado en el texto de Blackburne y Frank (2007). A diferencia de un micropanel, el cual posee una estructura de tiempo $(\mathrm{T})$ pequeña y las propiedades asintóticas de los estimadores están basadas en el límite cuando las observaciones de corte transversal (N) tienden a infinito, el macro panel tiene en consideración

3 Es un indicador significativo del poder de mercado potencial en la medida en que el comportamiento estratégico de las empresas es asimilable a una competencia en cantidades a la Cournot. Como lo establece Tirole (1988), el HHI dividido entre la elasticidad de la demanda es igual al índice de Lerner en el equilibrio de Cournot, es decir, el índice que mide la diferencia entre el precio de mercado y el coste marginaL.

4 Para la Comisión Europea, el nivel absoluto del hhi puede dar una indicación inicial de la presión competitiva en el mercado tras la concentración. Y una variación en el HHI es un indicador útil del cambio en el grado de concentración del mercado directamente derivado de una fusión. Es improbable que la Comisión detecte problemas de competencia horizontal en un mercado que después de la concentración tenga un HHI inferior a 1,000. Tampoco encuentra problemas de competencia horizontal en una concentración que dé lugar a un HHI entre 1,000 y 2,000 y una variación inferior a 250, o en una concentración que arroje un HHI superior a 2000 y una variación inferior a 150. Para más información ver Diario Oficial de la Unión Europea, 5.2.2004. p. 7. 
igualmente el crecimiento de la unidad de tiempo. En este caso particular, la propuesta metodológica se basa en una estimación de panel dinámico con dimensión T grande. Por tanto, asumiendo una estructura general del panel dinámico autorregresivo de retardos distribuidos tenemos la ecuación (1).

$$
y_{i t}=\sum_{j=1}^{p} \lambda_{i j} y_{i, t-j}+\sum_{j=0}^{q} \delta^{\prime}{ }_{i j} X_{i, t-j}+\mu_{i}+\epsilon_{i t}
$$

donde el número de empresas se designa por $i=1,2, \ldots, N$, el número de periodos por $t=1,2, \ldots, T, \delta_{i j}$ es un vector de coeficientes de $k \times 1, \lambda_{i j}$ son escalares, es el efecto específico por empresa, $\mathrm{y}_{i t}$ es la variable dependiente que en este caso es el logaritmo del Gap del PMS con respecto a los precios ofertados por cada una de las empresas y $X_{i t}$ es un conjunto de variables regresoras consideradas.

Ahora, dada la estructura general del modelo y con el objetivo de realizar el análisis de cointegración de largo plazo de las variables consideradas, es necesario realizar pruebas de raíz unitaria en panel y de cointegración, que indican la forma en la cual se debe estimar el modelo y si existe una relación de causalidad entre las variables de largo plazo. De esta forma, las pruebas de raíz unitaria indican si las variables son estacionarias o no.

En este caso específico se utilizan dos grupos de pruebas. El primer conjunto considera la independencia en los sucesivos cortes transversales de las series y están conformadas por las siguientes: LLC test (Levin, Lin \& Chu, 2002) y Breitung test (Breitung \& Das, 2005) que asumen una raíz unitaria para toda la serie, mientras que el IPS test (Im, Pesaran \& Shin, 2003) y el ADF-Fischer (Choi, 2001) admiten la existencia de diferentes raíces unitarias por medio de los cortes transversales. Por su parte, el Hadri test (Hadri, 2000), a diferencia de los anteriores, no construye la prueba bajo la hipótesis nula de raíz unitaria, sino que basa su test partiendo desde la estacionariedad de todas las series.

A continuación, las pruebas de cointegración desarrolladas se basan en los trabajos de Westerlund (2007) y Pedroni (1999). Donde en la primera no se impone un factor de restricción común para todas las series y plantea la prueba bajo la hipótesis de que no existe cointegración sobre las variables estudiadas. Mientras la segunda construye estadísticos paramétricos y no paramétricos, los cuales son normalizados para que distribuyan. Así, los tests están basados en probar si en un modelo de corrección de errores condicional, el término de corrección de error es igual a cero. En caso tal de rechazar la hipótesis nula se afirma que la correspondencia entre las variables del panel está cointegrada, lo que implica que las variables vuelven sobre sus relaciones de largo plazo a pesar de las dinámicas de corto plazo. 
Por otro lado, y retornando a la propuesta particular de este trabajo, si bien hay un conjunto de variables que influyen en el Gap del PMS, el eje central sobre el cual quiere hacer hincapié esta propuesta es sobre los efectos de la introducción de cambios regulatorios en el comportamiento del mercado. De esta forma, con el fin de recoger cada uno de estos efectos se introducirán dummies temporales, las cuales serán 1 cuando entra en vigencia la medida en el mercado y 0 en otro caso. Por lo tanto, a diferencia de la literatura convencional donde se hacen trabajos principalmente descriptivos, este trabajo se decanta por un análisis econométrico, lo cual ayuda a explicar cuáles son los efectos de los problemas regulatorios ${ }^{5}$.

En consecuencia, luego de testear la posible no estacionariedad de las series y la relación de cointegración, se evalúan cómo las desviaciones de la relación de equilibro de largo plazo de las variables son afectadas por dinámicas de corto plazo. Para esto se reparametriza la ecuación (1) mediante la especificación de un modelo de corrección de error de la siguiente forma:

$$
\Delta y_{i t}=\phi_{i}\left(y_{i, t-1}-\theta_{i}^{\prime} X_{i t}\right)+\sum_{j=1}^{p-1} \lambda_{i j}^{*} \Delta y_{i, t-1}+\sum_{j=0}^{q-1} \delta_{i j}^{\prime *} \Delta X_{i, t-j}+\mu_{i}+\epsilon_{i t}
$$

donde $\phi_{i}=-\left(1-\sum_{j=1}^{p} \lambda_{i j}\right), \quad \theta_{i}=\frac{\sum_{j=0}^{q} \delta_{i j}}{1-\sum_{k} \lambda_{i k}}, \quad \lambda_{i j}^{*}=-\sum_{m=j+1}^{p} \lambda_{i m}, \quad$ con $j=1,2, \ldots, p-1, \mathrm{y} \delta_{i j}^{*}=-\sum_{m=j+1}^{q} \delta_{i m}$ con $j=1,2, \ldots, q-1$. Así pues, es de esperarse que el parámetro de velocidad de ajuste $\varphi_{i}$ sea estadísticamente diferente de cero y significativamente negativo bajo el supuesto de que las variables retornan a su estado de equilibrio de largo plazo. Por su parte, el signo $\theta_{i}$ representa el vector de parámetros de las relaciones de largo plazo.

Entre las formas de estimación del modelo se encuentran las propuestas por Pesaran y Smith (1995) y Pesaran, Shin y Smith $(1997,1999)$. Así, en el modelo de la media del grupo (MG) los coeficientes del modelo son calculados mediante el promedio no ponderado del modelo heterogéneo sin restricciones, es decir, un promedio de las diferentes pendientes para cada una de las empresas. Otro tipo de estimación es la estimación pooled del modelo de la media del grupo (PMG), en el cual los efectos de largo plazo son restringidos para que sean iguales durante todo el panel. Por último, se tiene el modelo de efectos fijos dinámico donde todos los parámetros, excepto los interceptos,

5 Para realizar este trabajo se dispuso analizar cada uno de los Decretos y Reales Decretos vigentes en el mercado para el periodo propuesto, de los cuales solo los más importantes se consideran en la estimación del modelo. 
son restringidos para que sean iguales en todo el panel. De esta forma, se busca la cointegración de las variables, situación en la cual estas se ajustan a cualquier desviación de equilibrio de largo plazo.

Para contrastar la pertinencia tanto del modelo como de las variables elegidas se dispone de los datos suministrados por la página del OMI - Polo Español, S.A. (OMIE), quien es el operador del mercado responsable de la gestión económica del sistema. Esta página posee una plataforma ${ }^{6}$, la cual tiene acceso a datos históricos de precio marginal del sistema, ofertas, demanda y demás variables que comprenden el funcionamiento del mercado. Así, se tienen datos diarios del PMS, demanda, precios y cantidades de oferta para el periodo 2004-2006 por empresa.

En las figuras 1, 2 y 3 se muestran los logaritmos del Gap con el índice HHI y el RSI, además de la relación entre el PMS y el logaritmo de la demanda con el fin de observar la tendencia de cada una de estas series para el periodo de estudio.

En la figura 1 se observa cómo cada una de las empresas presenta en el logaritmo del Gap un comportamiento diferente, aspecto que se esperaba en la medida que cada una de ellas, a pesar de que operan en el mismo mercado, tienen estructuras de producción diferentes y, por lo tanto, se comportan de distinta manera a los incentivos propuestos. Si bien, cada empresa muestra un comportamiento volátil en el periodo analizado, este es más visible en el caso de Hidrocantábrico, Gas Natural y los Otros. No siendo lo mismo para Endesa e Iberdrola, donde esta última resalta por tener un poco más de tendencia que la primera. Por otra parte, no es claro si se presenta raíz unitaria para todas las series, en la medida que algunas se muestran relativamente estables, mientras que otras, como en el caso de Hidrocantábrico, muestran un claro cambio de tendencia para el último periodo.

En la figura 2 se evidencia la relación negativa entre el logaritmo del Gap y el RSI, manifestándose una especie de efecto espejo entre las variables, el cual es relevante especialmente para Enel, Hidrocantábrico y los Otros al final del periodo, sin considerar el cambio de nivel en marzo de 2006. Esto muestra cómo, a medida que se hace más necesaria la capacidad de producción de cada una de las empresas para abastecer la demanda, el Gap aumenta para cada una de ellas. Respecto a la estacionariedad del RSI, si bien para el primer periodo hay un comportamiento relativamente estable, el último periodo evidencia gran variabilidad, lo que podría estar indicando no estacionariedad. De esta forma, no es posible tener una conclusión clara respecto a la posible presencia de raíz unitaria.

6 Los datos son tomados a partir de la plataforma de OMIE, presentados a nivel horario y diario (OMIE, s.f.). 

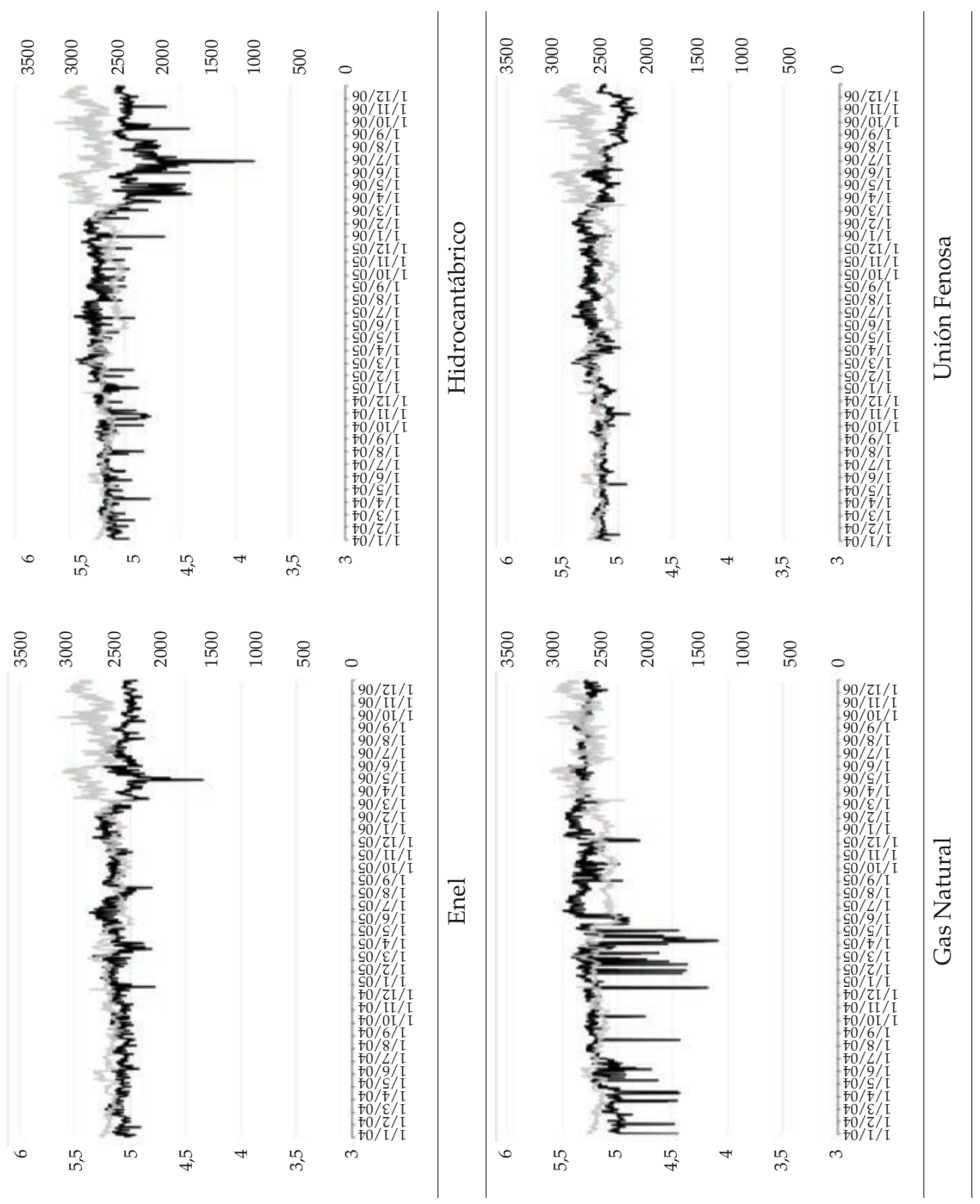


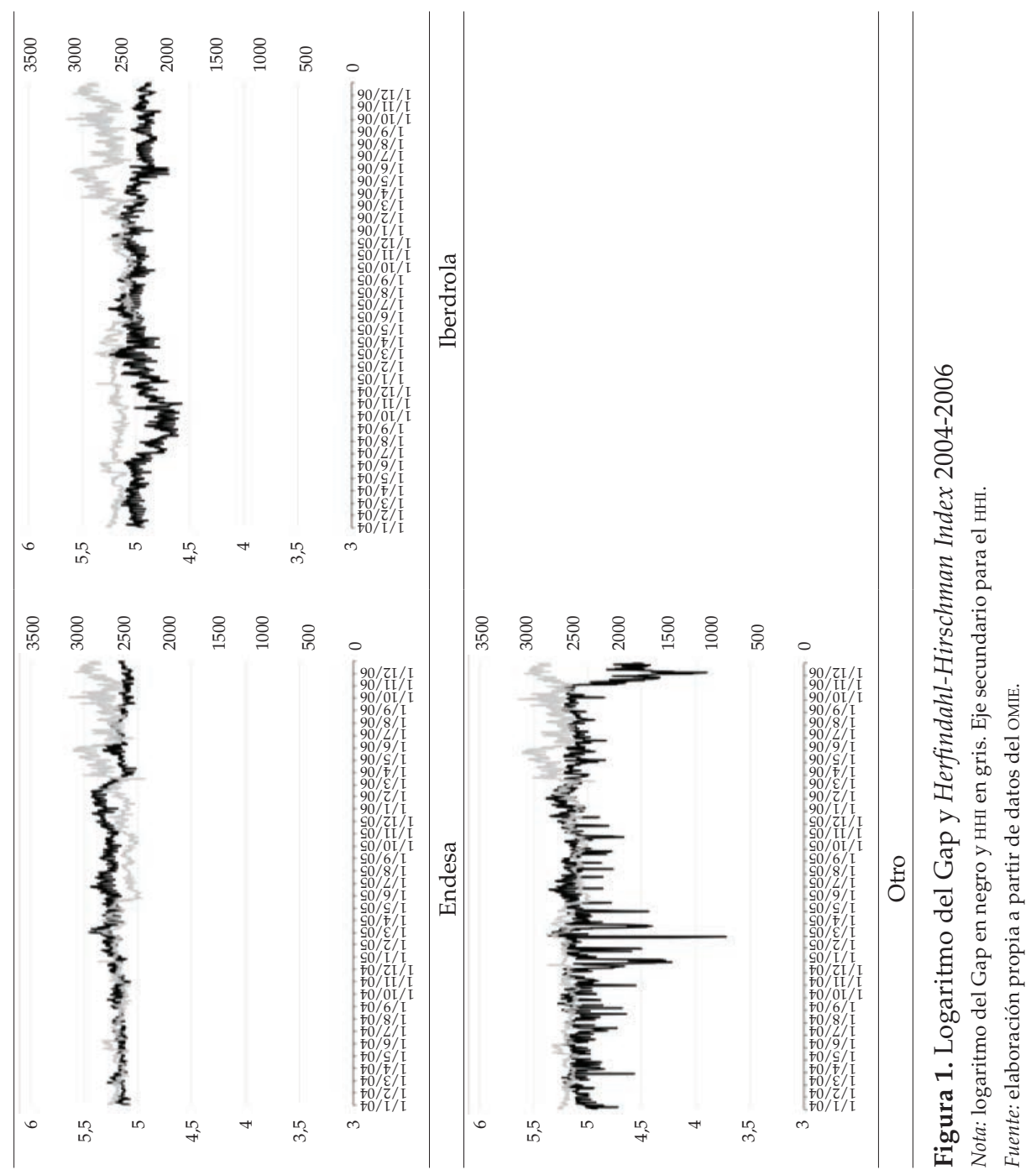



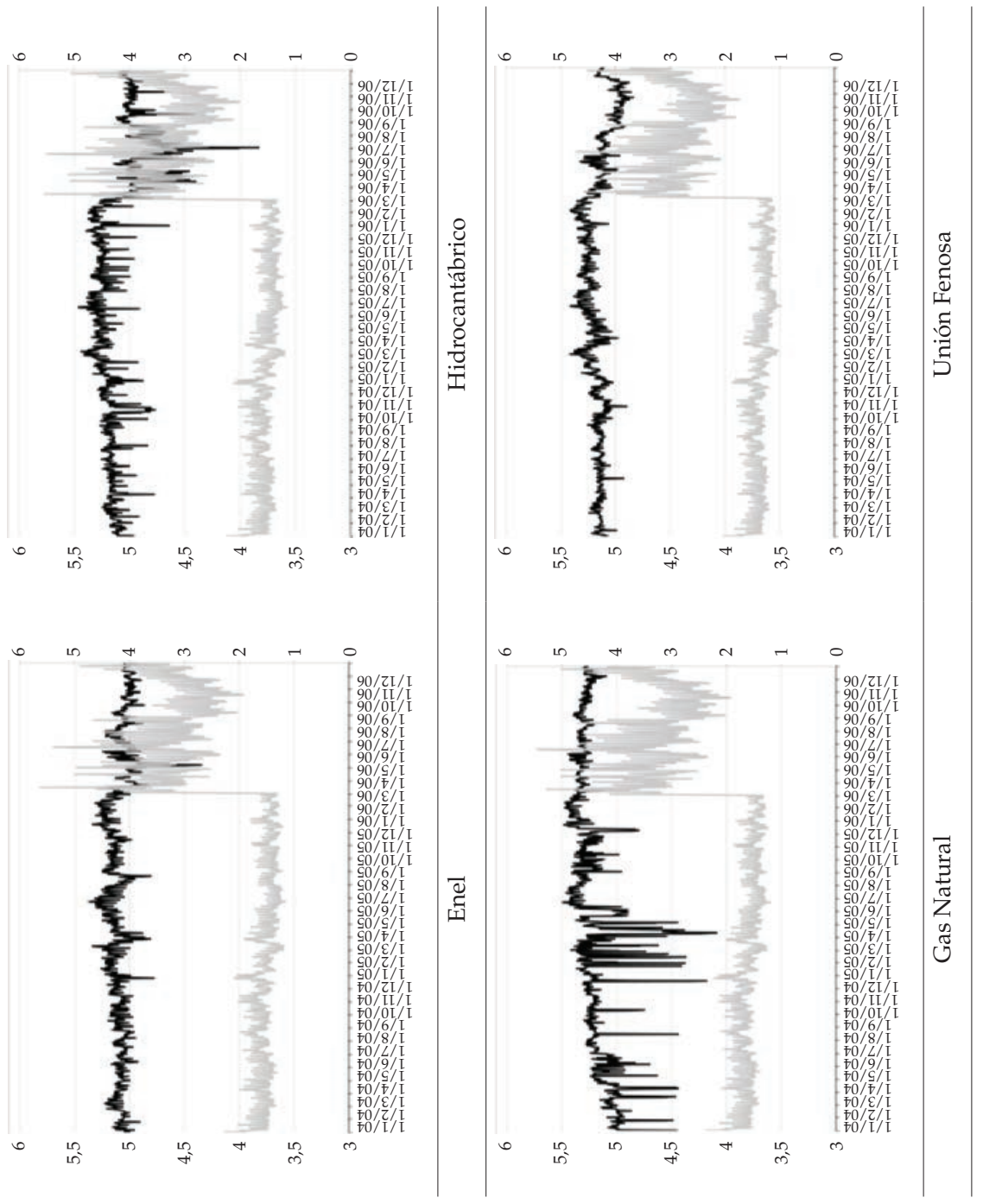


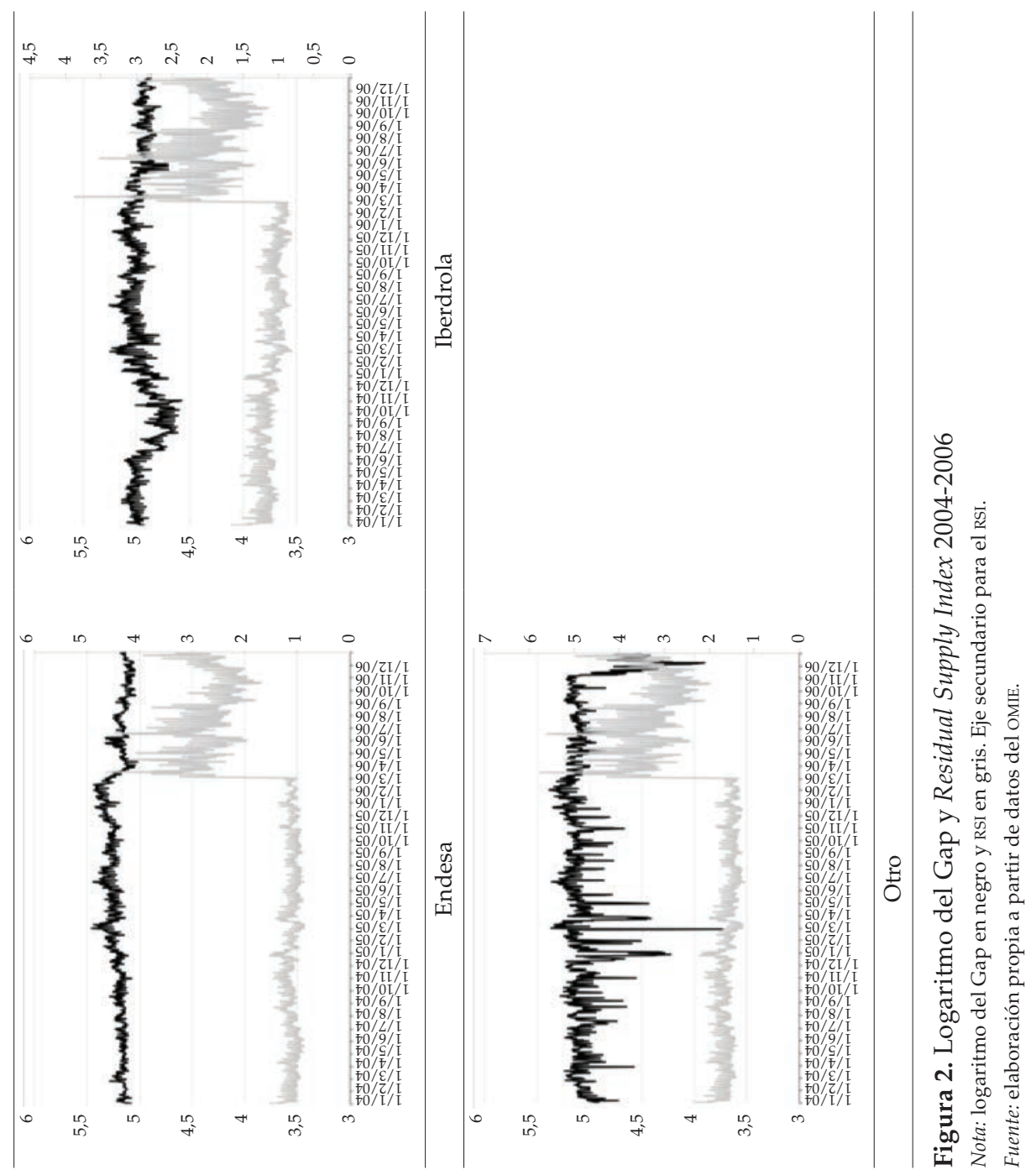


Por último, en la figura 3 se muestran el logaritmo de la demanda y el PMS. Se observa un crecimiento importante de la demanda en el primer tramo para luego disminuir después del cambio de nivel del PMS. Este resultado es paradójico en la medida en que el cambio se vio acompañado con una disminución de la demanda. No obstante, es de tener en cuenta que desde este momento se incluyeron cambios regulatorios importantes que pueden afectar la relación entre estas dos variables. Además, el comportamiento parece normalizarse al final del periodo. Con relación a la posibilidad de raíz unitaria, parece presentarse una especie de tendencia en la serie del log de la demanda, al igual que en el PMS.

A partir de esta descripción no es posible identificar claramente la estacionariedad de las series, la cual parece tener diferencias entre cada uno de los paneles y, por lo tanto, en la siguiente sección se presentan las pruebas formales de estacionariedad para esta estructura de datos de panel.

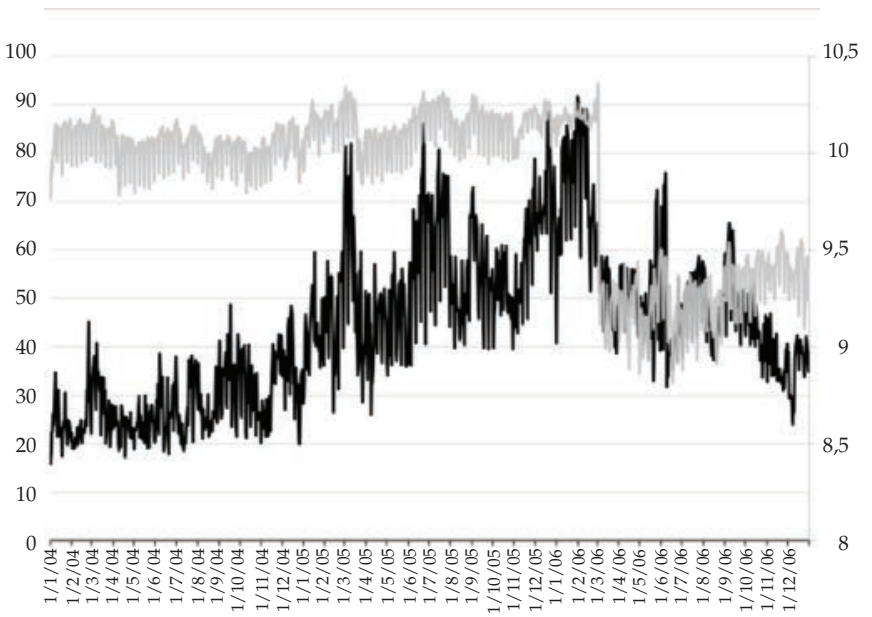

Figura 3. Logaritmo de la demanda y Precio Marginal del Sistema 2004-2006 Nota: PMS en negro y logaritmo de la demanda en gris. Eje secundario para el logaritmo de la demanda. Fuente: elaboración propia a partir de datos del omIE.

Finalmente, en la tabla 3 se pueden observar las principales variables consideradas en el modelo, incluyendo su definición y el signo esperado. 
Tabla 3. Variables consideradas en la regresión

\begin{tabular}{|c|c|c|}
\hline Variable & Definición & Signo esperado \\
\hline Gap del PMS & $\begin{array}{l}\text { Gap del precio marginal del sistema con respecto } \\
\text { al precio promedio ofertado por cada una de las } \\
\text { empresas }\end{array}$ & positivo \\
\hline Herfindahl-Hirschman (HHI) & Índice de concentración & positivo \\
\hline Residual Supply Index (RSI) & Capacidad de ejercer poder de mercado & negativo \\
\hline $\begin{array}{l}\text { Demanda promedio ho- } \\
\text { ra/día }\end{array}$ & Demanda promedio total horaria en el día & positivo \\
\hline PMS promedio hora/día & PMS promedio total horario en el día & positivo \\
\hline $\begin{array}{l}\text { Disponibilidad hidráulica } \\
\text { total }\end{array}$ & $\begin{array}{l}\text { Disponibilidad hidráulica total ofertada por las } \\
\text { empresas }\end{array}$ & negativo \\
\hline Índice general & Índice de Producción Industrial & positivo \\
\hline $\begin{array}{l}\text { Precio promedio tecnolo- } \\
\text { gía marginal }\end{array}$ & $\begin{array}{l}\text { Precio promedio de la tecnología marginal que en } \\
\text { este caso es el carbón para datos promedios diarios }\end{array}$ & positivo \\
\hline $\begin{array}{l}\text { Dummies de estructura } \\
\text { regulatoria }\end{array}$ & $\begin{array}{l}\text { Se incluirá una dummy cuando entre y/o se man- } \\
\text { tenga una nueva regla en el mercado y será cero en } \\
\text { otro caso }\end{array}$ & $\begin{array}{l}\text { positivo/ } \\
\text { negativo }^{7}\end{array}$ \\
\hline
\end{tabular}

Fuente: elaboración propia.

\section{Resultados empíricos}

A continuación, se profundiza en la presentación de los resultados descriptivos de las medidas de organización industrial, además de las pruebas de raíz unitaria en panel, cointegración, con la estimación de las relaciones propuestas para corto y largo plazo. Se finaliza con los resultados obtenidos a partir del cálculo del markup de beneficios.

\subsection{Medidas de organización industrial}

En el cálculo del RSI (figura 4) se puede observar cómo con la implementación del RDL 3/2006 se presenta una variación sustancial en el RSI para cada una de las empresas del pool. Para los años anteriores al cambio regulatorio se puede observar que tanto Endesa como Iberdrola son pivotes en varios días, lo que evidencia el poder de mercado que poseían estas dos empresas.

7 No se determina un efecto esperado claro, ya que esto dependerá de la particularidad de la norma. 
Esta variación es inclusive un poco más fuerte en los últimos meses antes de implementar la medida, lo que hace evidente la necesidad de adoptar correctivos que contrarresten la capacidad que tienen ambas empresas. Otro aspecto que se observa es la alta volatilidad del índice luego del cambio en las casaciones, lo que podría estar indicando que las empresas ajustan sus estrategias a las nuevas condiciones del mercado.

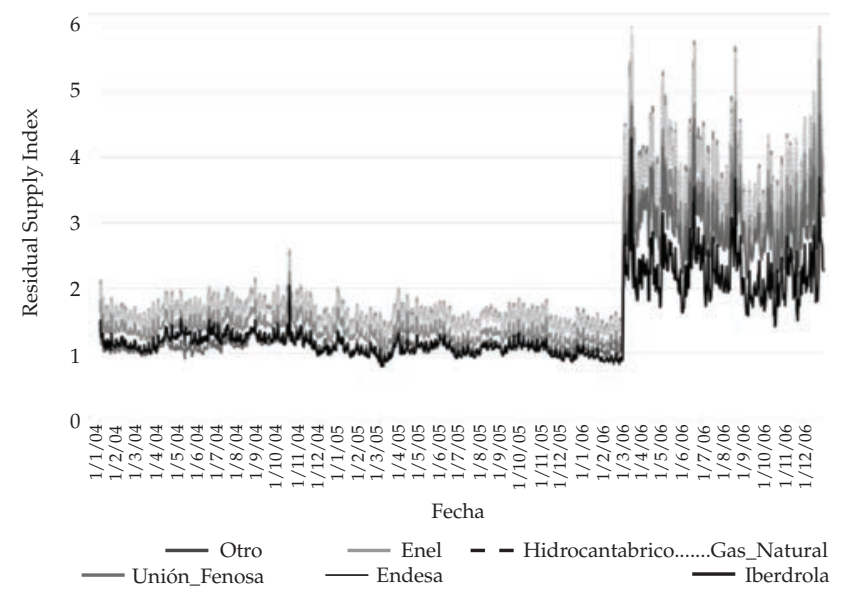

Figura 4. Residual Supply Index 2004-2006

Fuente: elaboración propia con base en datos de OMIE.

Con respecto al HHI, se puede observar claramente en la figura 5 una alta volatilidad del índice en cada uno de los días, siendo en todos los casos superior a 2200. Además, el cálculo presentado evidencia una tendencia a la baja en el nivel de concentración para el periodo posterior a marzo de 2006. No obstante, después de este año retorna a niveles mucho más altos presentados antes de la implementación de la medida. También, al igual que el caso del RSI, la varianza del índice aumenta considerablemente desde este momento.

\subsection{Pruebas de raíz unitaria en panel}

El anexo 1 presenta las pruebas correspondientes a la existencia de raíz unitaria en las series, incluyendo intercepto y tendencia. Así, el LLC rechaza la presencia de estacionariedad en las series del PMS, la disponibilidad hidráulica total, el índice de producción industrial y el precio promedio de la tecnología marginal. Por su parte, la prueba de Breitung acepta estacionariedad en las series. Con respecto a las pruebas que permiten un proceso autorregresivo diferente para cada corte como lo son IPS y Fisher, todas muestran estacionariedad. Por 


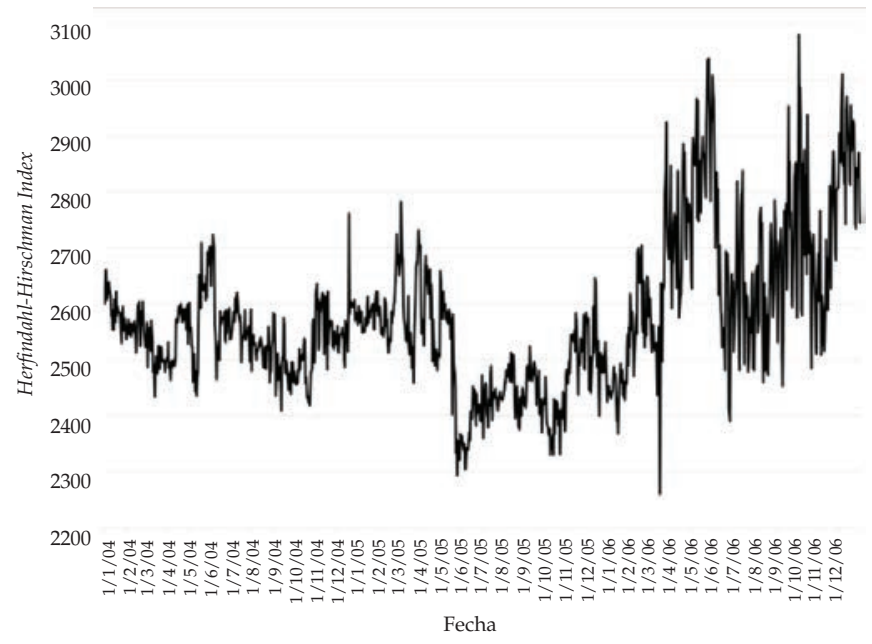

Figura 5. Herfindahl-Hirschman Index 2004-2006

Fuente: elaboración propia con base en datos de omIE.

otro lado, solo Hadri contradice la existencia de estacionariedad, a favor de la presencia de raíz unitaria en al menos uno de los paneles para todas las variables.

Por tanto, se puede concluir que no hay unificación de criterio en las pruebas. Esto podría presentarse por el hecho de que cada uno de los test concibe de forma diferente la existencia de raíz unitaria. Por ejemplo, en el caso de IPS y Fisher la hipótesis alternativa está vinculada al hecho de que al menos un panel es estacionario, mientras que en el caso de Hadri, la hipótesis nula es que todos los paneles son estacionarios. Dicho de este modo, las pruebas están indicando que algunos paneles son no estacionarios y otros sí. Por tanto, el panel contiene series estacionarias como no estacionarias, lo que hace pertinente realizar las pruebas de cointegración con el fin de mirar si existe una relación de equilibrio de largo plazo en las variables.

\subsection{Pruebas de cointegración}

Como se puede observar en el anexo 2, tanto en la prueba de Westerlund, incluyendo o no dependencia de corte transversal, como en el test de Pedroni se rechaza la hipótesis nula de no existencia de cointegración de las variables. Estas pruebas se realizaron incluyendo solo una de las tres variables de organización industrial para cada prueba. Así, se muestra la importancia de cada una de estas medidas en las relaciones de largo plazo de las variables 
del sistema. Por consiguiente, se tiene una relación de equilibrio de largo plazo entre el logaritmo del Gap y cada una de las variables consideradas en la regresión.

\subsection{Estimación e interpretación ${ }^{8}$}

La tabla 4 muestra las estimaciones teniendo en cuenta los efectos de largo plazo de los cambios regulatorios 1-2 y 1-3, correspondientes a las reglamentaciones RDL 3/2006, RDL 7/2006 y RDL 5/2005, respectivamente. Las estimaciones muestran para todos los casos que el parámetro de ajuste es negativo y estadísticamente significativo, con lo que se da cuenta que las relaciones de largo plazo, aquí presentadas se mantienen a pesar de las dinámicas de corto plazo. Por otro lado, se consideran simultáneamente dos dummies que tienen por objetivo recoger el efecto de la regulación en el logaritmo del Gap del precio marginal. Como se dijo previamente, la primera dummy quiere recoger el efecto del cambio en el mecanismo de casación en el mercado diario español, mientras la segunda, ya sea la dummy 2 o 3, indica el desmonte del mecanismo de recuperación de los стс.

Con respecto a las dummies 1 y 2, los resultados indican que el cambio en el mecanismo de casación tuvo un efecto positivo sobre el log del Gap, lo que podría interpretarse como un efecto nocivo sobre el mercado. Esto puede explicarse por la volatilidad que generó la medida. Además, tal y como afirma Fabra (2006), este tipo de medida no tiene los mismos incentivos derivados de una contratación bilateral en la disminución del precio, ya que las pocas empresas que entran en el mercado con posiciones netas vendedoras $y$, por lo tanto, las afecta el precio del mercado, no tienen la posición compradora en la producción cubierta mediante contratos financieros, lo que generaría una disminución en la remuneración de las unidades inframarginales.

Por su parte, el desmonte de los СтC se explica en el sentido contrario, ya que el signo positivo indica que mientras estuvo en vigencia, la regulación tenía efectos positivos sobre el Gap, aunque menores a los reportados por la primera medida considerada, es decir, el RDL 3/2006. Lo anterior es consistente con lo propuesto por Fabra y Toro (2005), Pérez-Arriaga et al. (2005) y Kühn y Machado (2004), quienes afirman cómo en los últimos años el efecto de los

8 Antes de realizar en análisis hay varios aspectos a tener en cuenta: primero, en el periodo de estudio no se desarrollaron medidas regulatorias en un mismo momento. Segundo, tampoco ocurrieron fusiones, compra o separación de empresas que pudieran afectar el objetivo del trabajo. Finalmente, se consideran separadamente HHI y RSI con el fin de evitar problemas de alta correlación de las variables independientes. 
CTC sobre el sistema no era tan beneficioso como en los primeros años de la medida. Así, la implementación de una medida con incentivos contrarios para las dos principales empresas, Endesa e Iberdrola, generó que se presentaran distorsiones en el mercado.

Por otro lado, las dummies 1 y 3 hacen alusión a la modificación en el mecanismo de casación y el aplazamiento del pago de los CTC correspondientes del año 2004 al 2006, respectivamente. En general, las estimaciones muestran el mismo efecto de la primera regulación, donde el cambio tuvo un efecto positivo sobre el Gap. El aplazamiento en el pago de los CTC va en línea con su posterior desmonte en 2006. Por tanto, a pesar que esta medida fue incorporada como una solución a los problemas que tenía el mercado en este periodo, incrementan la poca credibilidad en el mecanismo de CTC (Federico, Vives \& Fabra, 2008).

Así los resultados muestran lo encontrado por otros autores, los cuales afirmaban cómo, desde varios meses antes de su derogación, la medida de los CTC no tenía sino efectos distorsionadores sobre el mercado y ya no actuaba como un price cap. Uno de los aspectos que fundamenta esta afirmación es que en este caso el cambio regulatorio (dummy 3) parece ser estadísticamente más relevante que el desmonte formal de los CTC (dummy 2), siendo temporalmente anterior. De esta forma, el resultado implica que el real efecto positivo que tenían los СТC sobre el sistema se diluyó mucho antes que su desmonte formal. En resumen, los cálculos muestran que el cambio en la casación generó un crecimiento entre 6,3 \% y 13,8 \% en la mediana del Gap del PMS. Por su parte, el desmonte de los CTC causó una reducción entre 1,2 \% y $5,4 \%$ en la misma variable, ya sea si se considera desde la derogación formal de la medida o el aplazamiento anterior de los pagos.

Además, los resultados de largo plazo con respecto al logaritmo de la demanda rezagado, el Precio Marginal del Sistema (PMS) rezagado y las medidas del HHI O RSI ${ }^{9}$ también se incluyen en las tablas anteriores. Como era de esperarse, se encuentra una relación positiva entre la demanda y el Gap del precio marginal entre 0,11 a 0,44 . Esto se puede explicar claramente ya que la demanda ejerce presión sobre los precios, lo que genera que las empresas tengan mayor capacidad de maniobra en la determinación del precio, especialmente si se tiene en cuenta que la demanda de electricidad es generalmente un bien inelástico (Labandeira et al., 2012).

9 Inicialmente también se consideró incluir en el análisis el índice de Demanda Residual, pero no mostró resultados importantes a lo ya reportado por el RSI. Además, este último es ampliamente utilizado en mercados como California (Twomey, et al, 2006). 


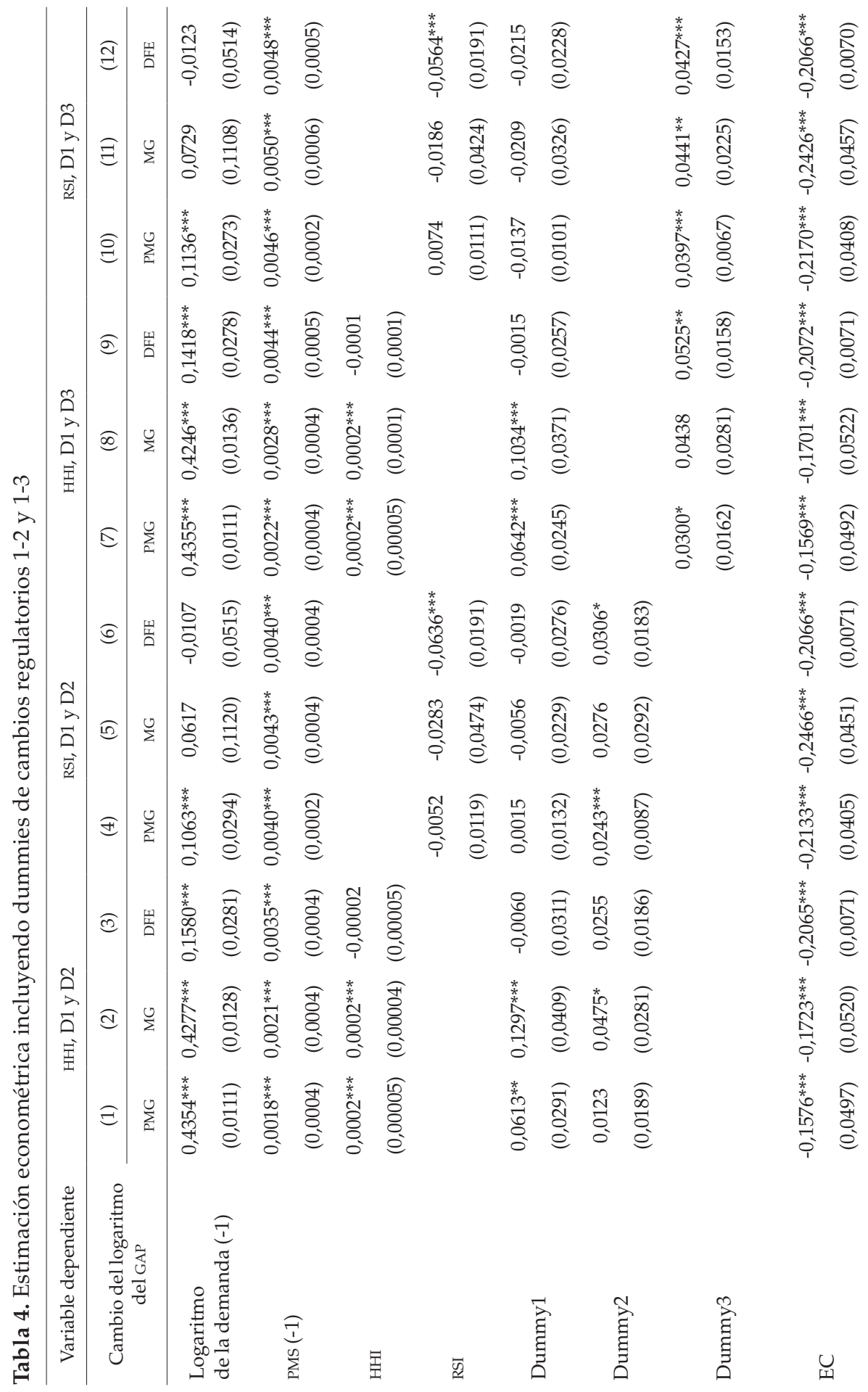




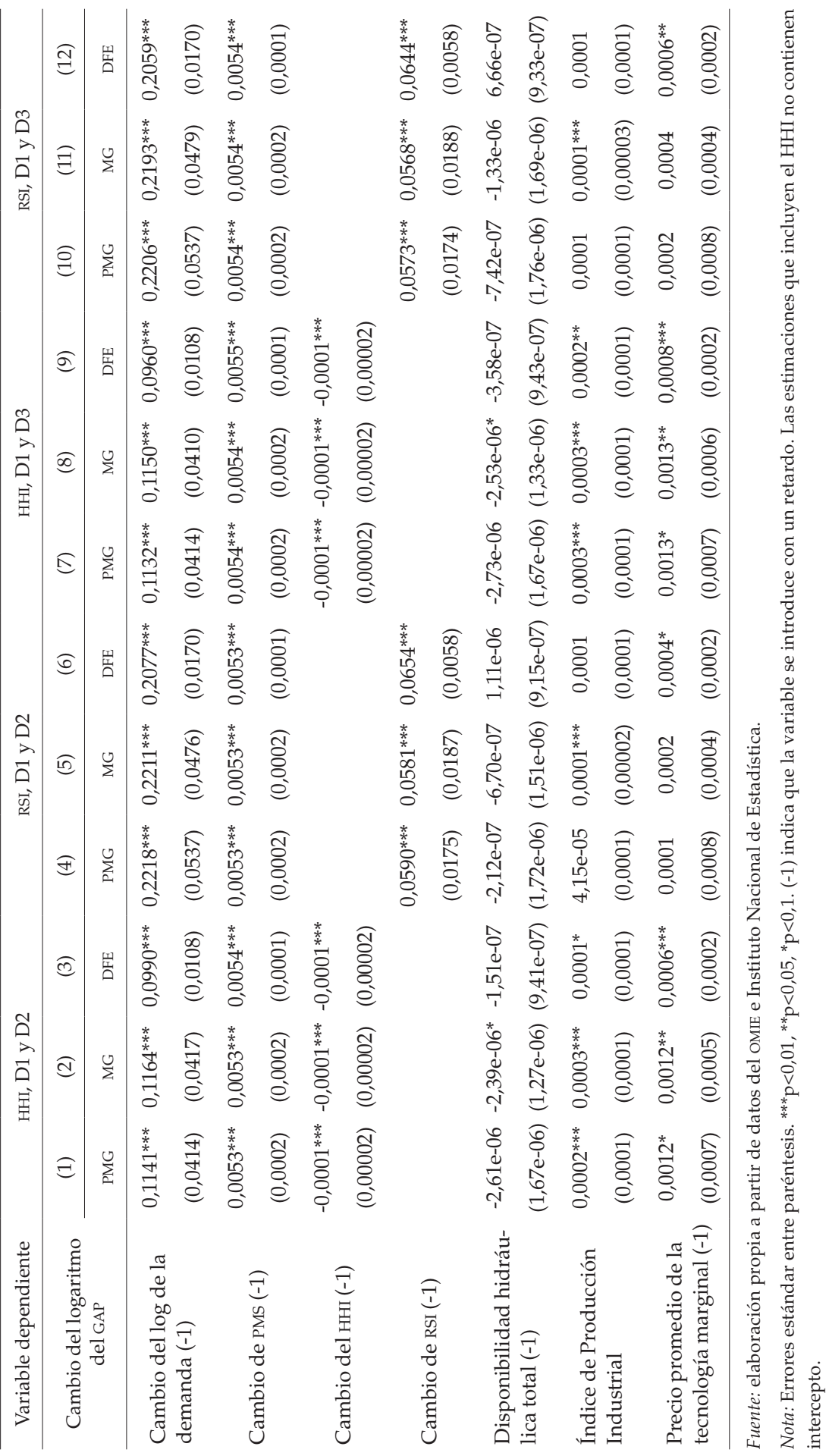


Por su parte, el efecto del PMS es fácilmente explicable desde la definición del Gap utilizada en este estudio. En cuanto a las medidas de organización industrial, es evidente que a mayor concentración del mercado (HHI), las empresas tengan una mayor oportunidad de ejercer poder de mercado, hecho claro en las estructuras oligopolísticas (Belleflamme \& Peitz, 2010; Cabral, 2000). Además el RSI, el cual es una medida directa de la capacidad de ejercer poder de mercado identificando agentes pivotes funciona de manera inversa, presenta los resultados esperados, aunque su significancia estadística no sea tan persistente.

En cuanto a los resultados de corto plazo, en este caso se tuvieron en cuenta el cambio en cada una de las variables de largo plazo más la disponibilidad hidráulica rezagada, el índice de producción industrial y el precio ofertado promedio de la tecnología marginal, que en este caso es carbón. En primer lugar, se encuentran los resultados esperados, tanto para el cambio de la demanda como la de PMS, no obstante, en referencia al HHI y RSI, el cambio tiene signo contrario al esperado y estadísticamente significativo para cada una de las estimaciones. Lo anterior podría estar indicando que la intención de obtener beneficios extraordinarios por parte de las empresas es analizada por ellas como una estrategia de largo plazo, más que de corto plazo. En segundo lugar, las demás variables de oferta, como disponibilidad hidráulica y el precio promedio de la tecnología marginal, muestran resultados acordes a lo que establece la teoría económica - negativo y positivo - respectivamente. Igualmente, en el caso de la variable de demanda considerada, el índice de producción industrial, el cual puede considerarse indirectamente como una medida de presión de la demanda por electricidad.

\section{Conclusiones}

El trabajo muestra cómo los sucesivos cambios regulatorios acontecidos en el periodo 2004-2006 en el pool eléctrico español eran decisivos para un buen funcionamiento de este mercado. El desmonte tardío del sistema de стс le costó al sistema un aumento en la mediana del Gap del pMS entre 1,2\% y 5,4\%, mientras que el cambio en la casación de las ofertas presentó un aumento entre el $6,3 \%$ a $13,8 \%$ en la misma variable. Por lo tanto, según este estudio, el desmonte de los стс tuvo un efecto beneficioso menor al resultado negativo del cambio en las casaciones, lo que a su vez ha conllevado a que este mercado tenga una de las tarifas más altas a nivel mundial. Además, esta investigación se destaca porque incluye medidas de organización industrial para la explicación del comportamiento del mercado de electricidad en 
España, conjuntamente con la modelación explícita de los efectos de cambios regulatorios ordenados por las autoridades. Así, se muestran empíricamente cómo las relaciones teóricas sobre aspectos como concentración del mercado y la capacidad de ejercer poder de mercado son verificadas en la industria. Específicamente, se evidencia para los años anteriores a la implementación del cambio regulatorio que Endesa e Iberdrola son pivotes.

Como era de esperarse, se encuentra una relación positiva entre la demanda y Gap del precio marginal, lo cual se puede explicar a que la demanda ejerce presión sobre los precios, lo que hace que las empresas puedan tener mayores comportamientos estratégicos para la fijación de precios, máxime cuando se trata de un bien necesario como es el servicio de electricidad. Respecto a variables de oferta como la disponibilidad hidráulica y el precio promedio de la tecnología marginal, presentan resultados acordes con la teoría económica, negativo y positivo respectivamente.

Finalmente, sería importante ampliar el periodo de estudio con el fin de incluir otras medidas relevantes para el funcionamiento del mercado, que tal y como se observa en la actualidad, quizás pudieran ayudar a explicar el alto déficit por el cual atraviesa el mercado eléctrico español.

\section{Referencias}

Agosti, L., Padilla, J. A., \& Requejo, A. (2007). El mercado de generación eléctrica en España: estructura, funcionamiento y resultados. Economía industrial 364, 21-37.

Belleflamme, P., \& Peitz, M. (2010). Industrial organization: markets and strategies. Cambridge University Press: Cambridge.

Blackburne, E. F., \& Frank, M. W. (2007). Estimation of nonstationary heterogeneous panels. Stata Journal, 7(2), 197.

Breitung, J., \& Das, S. (2005). Panel unit root tests under cross sectional dependence. Statistica Neerlandica, 59(4), 414-433.

Cabral, L. M. B. (2000). Introduction to industrial organization. MIT Press: Cambridge.

Ciarreta, A., \& Espinosa, M. P. (2012). The impact of regulation on pricing behavior in the Spanish electricity market (2002-2005). Energy Economics, 34(6), 2039-2045.

CNE. (2005). Informe de la Comisión sobre el Proyecto de Concentración Consistente en la Adquisición del Control de Endesa, S.A. por parte de Gas Natural SDG, S.A. Mediante Oferta Pública de Adquisición de Acciones. Aprobado por el Consejo de Administración de 20 de diciembre de 2005. 
CNE. (2007). Exposición y Análisis de un Caso: Concentración/Fusión/Adquisición de Empresas. Recuperado de https://int.search.tb.ask.com/ search /GGmain.jhtml? searchfor $=$ Exposici $\%$ C3\%B3n+y+An $\%$ C3\% A1lisis+de+un+Caso $\% 3 \mathrm{~A}+\mathrm{Concentraci} \% \mathrm{C} 3 \% \mathrm{~B} 3 \mathrm{n}+\mathrm{Fusi} \% \mathrm{C} 3 \% \mathrm{~B} 3 \mathrm{n}+$ Adquisici $\%$ C3\%B3n+de+Empresas + CNE\&st $=$ sb\&tpr $=0$ mni\&p2 $=\%-$ 5EY6\%5Expu034\%5ELMES\%5Eco\&ptb=90B58796-C4A4-4690-9BCB-593 5201761EB\&n=783a5e21\&si=adwords-8ef3fceeefcfb6200a162c4345a2fc37

Choi, I. (2001). Unit root tests for panel data. Journal of international money and Finance, 20(2), 249-272.

De Frutos, M. A., \& Fabra, N. (2008). On the impact of forward contract obligations in multi-unit auctions. CEPR Discussion Paper.

Fabra, N. (2006). El Funcionamiento del Mercado Eléctrico Español bajo la Ley del Sector Eléctrico. En Comisión Nacional de Energía (Editor), Energía: del monopolio al mercado. Editorial Aranzadi, S. A.: Madrid.

Fabra, N., \& Toro, J. (2005). Price wars and collusion in the Spanish electricity market. International Journal of Industrial Organization, 23(3), 155-181.

Federico, G., Vives, X., \& Fabra, N. (2008). Competition and regulation in the Spanish gas and electricity markets. Report of the Public-Private Sector Research Center, Navarra: IESE Business School.

García-Díaz, A., \& Marín, P. L. (2003). Strategic bidding in electricity pools with short-lived bids: an application to the Spanish market. International Journal of Industrial Organization, 21(2), 201-222.

Gianfreda, A., \& Grossi, L. (2012). Forecasting Italian electricity zonal prices with exogenous variables. Energy Economics, 34(6), 2228-2239.

Green, R. J., \& Newbery, D. M. (1992). Competition in the British electricity spot market. Journal of political economy, 5(100), 929-953.

Hadri, K. (2000). Testing for stationarity in heterogeneous panel data. The Econometrics Journal, 3(2), 148-161.

Im, K. S., Pesaran, M. H., \& Shin, Y. (2003). Testing for unit roots in heterogeneous panels. Journal of econometrics, 115(1), 53-74.

Kuhn, K. U., \& Machado, M. P. (2004). Bilateral market power and vertical integration in the Spanish electricity spot market. CEPR Discussion Paper.

Labandeira, X., Labeaga, J. M., \& López-Otero, X. (2012). Estimation of elasticity price of electricity with incomplete information. Energy Economics, 34(3), 627-633.

Levin, A., Lin, C.-F., \& Chu, C.-S. J. (2002). Unit root tests in panel data: asymptotic and finite-sample properties. Journal of econometrics, 108(1), 1-24.

Moutinho, V., Moreira, A. C., \& Mota, J. (2014). Do regulatory mechanisms promote competition and mitigate market power? Evidence from Spanish electricity market. Energy Policy, 68, 403-412. 
Ocaña, C., \& Romero, A. (1998). Una simulación del funcionamiento del pool de energía eléctrica en España. CNSE, DT 002/98, 1-57. Recuperado de http: / / docplayer.es /14724070-Una-simulacion-del-funcionamiento-del-poolde-energia-electrica-en-espana.html.

OMI - Polo Español. Base de datos. Recuperado de http:/ / www.omie.es / files / flash/ResultadosMercado.swf.

Pedroni, P. (1999). Critical values for cointegration tests in heterogeneous panels with multiple regressors. Oxford Bulletin of Economics and statistics, 61(S1), 653-670.

Pérez Arriaga, J. I., Batlle, C., Vázquez, C., Rivier, M., \& Rodilla, P. (2005). Libro Blanco sobre la reforma del marco regulatorio de la generación eléctrica en España. Madrid: Minetad. Recuperado de http:/ / www.minetad.gob. es/energia/electricidad/regimenordinario/documents/libroblanco.pdf Pesaran, M. H., Shin, Y., \& Smith, R. P. (1997). Estimating long-run relationships in dynamic heterogeneous panels. DAE Working Papers Amalgamated Series 9721.

Pesaran, M. H., Shin, Y., \& Smith, R. P. (1999). Pooled mean group estimation of dynamic heterogeneous panels. Journal of the American Statistical Association, 94(446), 621-634.

Pesaran, M. H., \& Smith, R. (1995). Estimating long-run relationships from dynamic heterogeneous panels. Journal of econometrics, 68(1), 79-113.

Tirole, J. (1988). The theory of industrial organization. MIT Press: Cambridge.

Twomey, P., Green, R., Neuhoff, K., \& Newbery, D. (2006). A Review of the Monitoring of Market Power. CMI Working Paper.

Von der Fehr, N. H. M., \& Harbord, D. (1993). Spot market competition in the UK electricity industry. The Economic Journal, 103(418), 531-546.

Westerlund, J. (2007). Testing for error correction in panel data. Oxford Bulletin of Economics and statistics, 69(6), 709-748. 


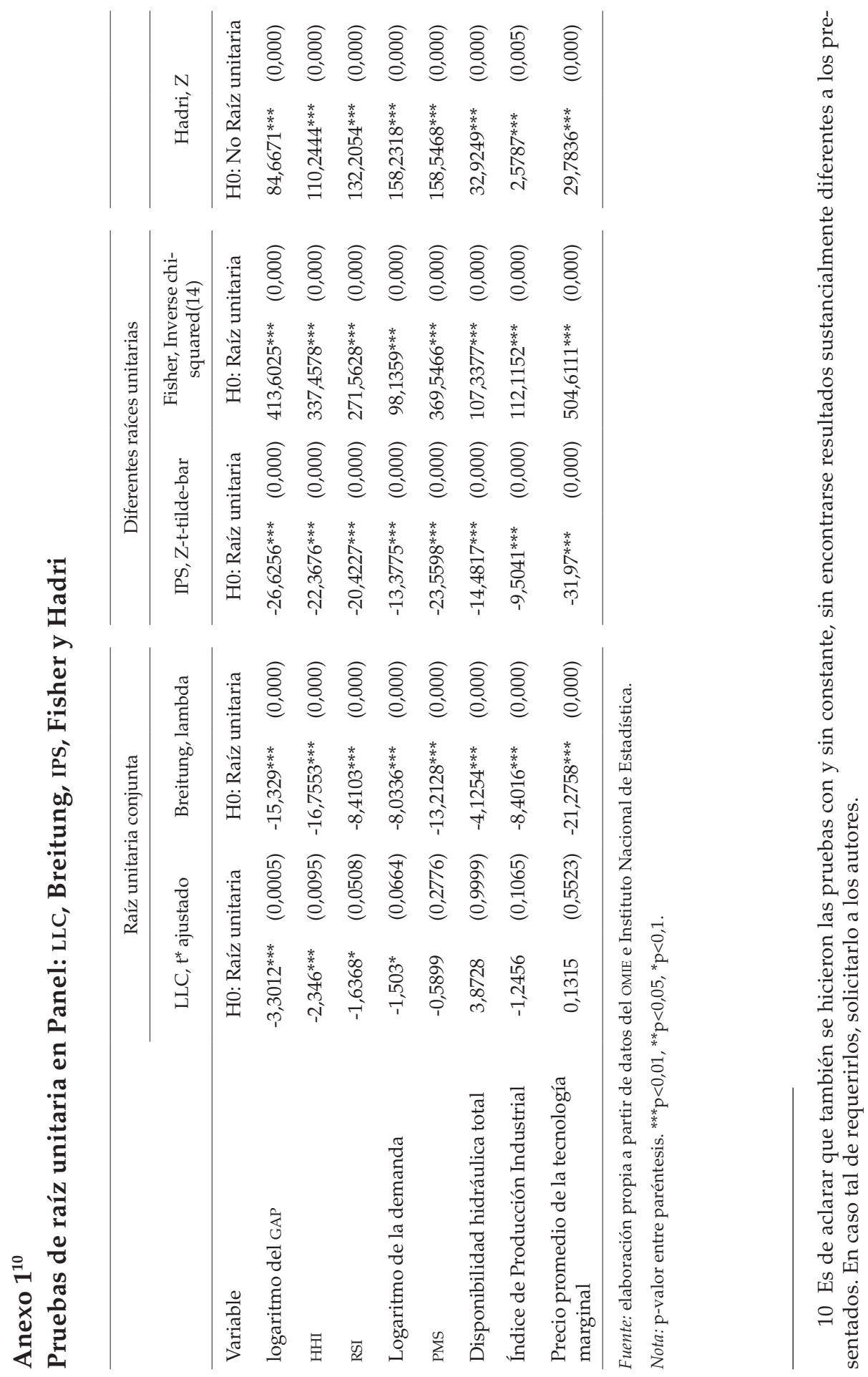




\section{Anexo $2^{11}$}

\section{Pruebas de Cointegración: Westerlund y Pedroni}

\begin{tabular}{lccccc}
\hline \multicolumn{1}{c}{ Prueba } & $\begin{array}{c}\text { Variable de Organización } \\
\text { Industrial incluida }\end{array}$ & Valor & Valor z & P-valor & P-valor Robusto \\
\hline $\mathrm{Gt}$ & & $-8,323$ & $-14,7$ & $(0,000)$ & $(0,000)$ \\
$\mathrm{Ga}$ & $-142,031$ & $-32,96$ & $(0,000)$ & $(0,000)$ \\
$\mathrm{Pt}$ & $\mathrm{HHI}$ & $-24,963$ & $-17,236$ & $(0,000)$ & $(0,000)$ \\
$\mathrm{Pa}$ & & $-165,157$ & $-40,857$ & $(0,000)$ & $(0,000)$ \\
$\mathrm{Gt}$ & $-8,588$ & $-15,456$ & $(0,000)$ & $(0,000)$ \\
$\mathrm{Ga}$ & & $-150,378$ & $-35,238$ & $(0,000)$ & $(0,000)$ \\
$\mathrm{Pt}$ & $\mathrm{RSI}$ & $-25,395$ & $-17,671$ & $(0,000)$ & $(0,000)$ \\
$\mathrm{Pa}$ & & $-171,663$ & $-42,659$ & $(0,000)$ & $(0,000)$ \\
\hline
\end{tabular}

\begin{tabular}{|c|c|c|c|}
\hline & & & \\
\hline Panel-v & & 13,79 & $(0,000)$ \\
\hline Panel-rho & & $-47,82$ & $(0,000)$ \\
\hline Panel-t & & $-26,36$ & $(0,000)$ \\
\hline Panel-adf & HHI & $-3,898$ & $(0,000)$ \\
\hline Group-rho & & $-61,83$ & $(0,000)$ \\
\hline Group-t & & $-31,65$ & $(0,000)$ \\
\hline Group-adf & & $-6,223$ & $(0,000)$ \\
\hline Panel-v & & 14,62 & $(0,000)$ \\
\hline Panel-rho & & $-55,32$ & $(0,000)$ \\
\hline Panel-t & & $-29,08$ & $(0,000)$ \\
\hline Panel-adf & RSI & $-5,29$ & $(0,000)$ \\
\hline Group-rho & & $-68,24$ & $(0,000)$ \\
\hline Group-t & & $-34,43$ & $(0,000)$ \\
\hline Group-adf & & $-7,464$ & $(0,000)$ \\
\hline
\end{tabular}

Fuente: elaboración propia a partir de datos del OMIE e Instituto Nacional de Estadística.

$11 \mathrm{Al}$ igual que en caso anterior, se hicieron las pruebas con y sin constante para la prueba de Westerlund y solo constante para la de Pedroni, se encontró cointegración en todas las opciones. En caso tal de requerirlos, solicitarlo a los autores. 
